\title{
NOUVELle
}

\section{Approche personnalisée des conditionnements pré- transplantation de patients porteurs de syndromes myélodysplasiques}

Marie Goulard $^{1}$, Christine Dosquet ${ }^{1,2}$, Christine Chomienne ${ }^{1,2}$
${ }^{1}$ Inserm UMR-S-1131, 1, avenue Claude Vellefaux, 75010 Paris, France.

${ }^{2}$ APHP, Hôpital Saint-Louis, unité de biologie cellulaire, 1, avenue Claude Vellefaux, 75010 Paris, France. cchomienne@institutcancer.fr
> Les syndromes myélodysplasiques (SMD) constituent un groupe hétérogène de pathologies myéloïdes clonales caractérisées par une hématopoïèse inefficace, des cytopénies sanguines et un risque de progression vers des leucémies myéloïdes aiguës (LAM). Les SMD représentent les plus fréquents états pré-leucémiques de l'adulte. Leur incidence a augmenté au cours des dernières années, principalement chez les personnes âgées, avec une médiane, au diagnostic, de 70 ans [1]. L'origine supposée des SMD est une transformation des cellules souches/progéniteurs hématopoïétiques, présentant des anomalies génétiques et cytogénétiques [2]. Chez les enfants et les jeunes adultes atteints de SMD, des mutations congénitales sont observées. Le déclenchement des SMD peut également être secondaire à un traitement anti-cancéreux antérieur, ou à une exposition à des produits toxiques, comme le benzène ou les pesticides [3]. Cependant, dans la plupart des cas, les SMD surviennent en l'absence d'exposition à des cytotoxiques ou de facteurs prédisposants. Les SMD sont classés selon le score IPSS (international prognostic scoring system) en fonction de leur pronostic: bas risque, risque intermédiaire et haut risque. La prise en charge des patients atteints de SMD repose sur un traitement symptomatique des cytopénies. La greffe allogénique de cellules souches hématopoïétiques (CSH) est le seul traitement curatif, mais les risques de mortalité après greffe demeurent élevés. Des mutations génétiques sont associées au phénotype clinique des patients. L'équipe de BL Ebert a ainsi étudié le rôle prédictif potentiel de mutations génétiques sur la réussite de la transplantation de CSH (traduite par la médiane de survie) chez les patients atteints de SMD [4]. Cette étude a été réalisée à partir de la biobanque et de la base de données cliniques du CIBMTR (Center for international blood and marrow transplant research). Les échantillons biologiques et les données cliniques de 1514 patients atteints de SMD provenant de 130 centres de greffes ont été analysés. L'étude génétique des prélèvements réalisés lors du préconditionnement ${ }^{1}$, a consisté au séquençage de 129 gènes connus pour être impliqués dans l'oncogenèse de cancers myéloïdes, ou caractéristiques d'une insuffisance médullaire.

\section{Mutations des SMD et survie des patients}

Dans la cohorte de patients examinés, les gènes driver les plus fréquemment mutés sont ceux qui avaient été décrits dans cette population majoritairement à haut risque, qui bénéficie d'une transplantation de CSH. II s'agit, par ordre décroissant de fréquence supérieure ou égale à $10 \%$, des gènes $A S X L 1$

1 Étape préalable à la greffe consistant en un traitement immunodépresseur. (additional sex comb-like 1), TP53 (suppresseur de tumeur p53), DNMT3A (DNA méthyltransférase 3A), TET2 (tet methylcytosine dioxygenase 2), RUNXI (Runt-related transcription factor), et SF3B1 (splicing factor $3 b$ subunit 1). Les patients classés à haut risque présentent fréquemment des mutations des gènes TP53 et DNMT3A. Les mutations du gène SF3BI sont par contre observées chez les patients présentant un risque plus faible. De nombreuses variables interviennent dans la survie post-transplantation. Un âge élevé (supérieur à 40 ans), un score Karnofsky ${ }^{2}$ en dessous de 90, une incompatibilité HLA (human leucocyte antigen), un pourcentage élevé de blastes dans la moelle osseuse, une thrombocytopénie sévère et un caryotype complexe sont associés à une survie courte. Au contraire, une anémie aplasique et une hémoglobinurie paroxystique nocturne sont reliées à une survie plus longue du patient.

Certaines mutations sont directement associées à la survie du patient après transplantation. Ainsi, une mutation du gène TP53 ou des gènes de la voie de signalisation $\operatorname{Ras}^{3}$ (NRAS, KRAS, PTPN11,

\footnotetext{
${ }^{2}$ Index conçu en 1949 par Sloan Kettering, qui décrit l'état général du patient.

${ }^{3}$ Les gènes RAS codent une famille de protéines membranaires liant le GTP et qui sont impliquées dans la régulation de la croissance cellulaire. Quelques exemples: NRAS: neuroblastoma RAS viral oncogene homolog; KRAS: v-Kiras2 Kirsten rat sarcoma viral oncogen homolog; PTPN11: tyrosine-protein phosphatase non-receptor type 11; NFI: neurofibromatosis type 1; RIT1: Ras-like without CAAX 1; FLT3 : FMS-like tyrosine kinase 3 ; Kit : tyrosine kinase, CD117.
} 


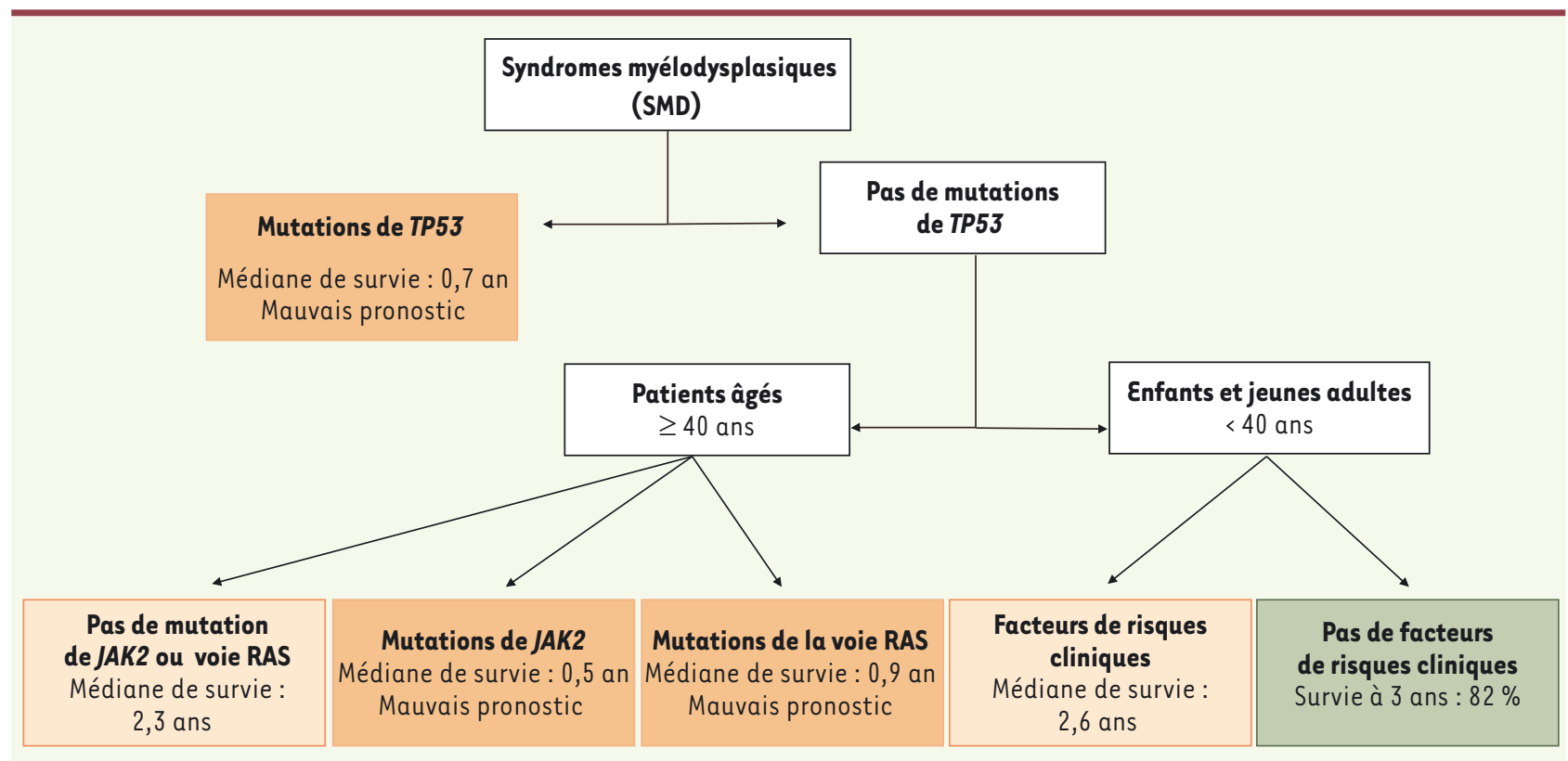

Figure 1. Modèle de partitionnement récursif de la survie des patients atteints de SMD en fonction des facteurs cliniques, génétiques et du conditionnement pré-transplantation. Les patients atteints de syndrome myélodysplasique (SMD) présentant des mutations de la voie de signalisation Ras sont les seuls à bénéficier d'un conditionnement intensif myéloablatif. Les facteurs de risques cliniques sont les SMD secondaires, le nombre de plaquettes et le pourcentage de blastes dans la moelle au diagnostic.

CBL, NFl, RITI, FLT3 et KIT) est associée à une courte survie du patient et à une rechute rapide après transplantation. La survie du patient est d'autant plus courte que TP53 est tronquée, ceci résultant d'une mutation antisens. Les SMD secondaires ont une survie plus faible après transplantation que les SMD primaires. Chez ces patients, TP53 et son régulateur PPMID $\left(\mathrm{Mg}^{2+} /\right.$ $\mathrm{Mn}^{2+}$-dependent protein phosphatase 1D) sont plus fréquemment mutés. Les mutations de TP53 (localisé en 17p) sont associées à des caryotypes de haut risque et entraînent des résistances aux thérapies conventionnelles. Le taux de mutations de TP53 est directement lié à la survie du patient et ce gène se révèle être une cible thérapeutique de choix dans les SMD et les LAM [5]. La mutation V617F (valine en position 617 mutée en phénylanaline) du gène codant JAK2 (janus kinase 2) est également associée à une survie courte et à un haut taux de rechute. Aucune mutation n'a pu être reliée à une survie prolongée.

Les mutations de TET2, DNMT3A, SRSF2, $S F 3 B I$ et PPMID sont plus communes chez les patients âgés que chez les enfants et les jeunes adultes (de moins de 40 ans) $[6,7](\rightarrow)$.

Les mutations

$(\rightarrow)$ Voir la Nouvelle de 0 . Bernard et al., $m / s n^{\circ} 3$, mars 2015, page 268 et la Synthèse de G. Dujardin et al., $\mathrm{m} / \mathrm{s}$ $n^{\circ} 12$, décembre 2016, page 1103 touchant GATA2 (family of zinc-finger transcription factors), PIGA (phosphatidylinositol $\mathrm{N}$-acetylglucosaminyltransferase subunit $A$ ) et des mutations hétérozygotes du gène SBDS (Shwachman-bodian diamond syndrome) sont les trois mutations les plus communes chez les patients de moins de 40 ans. Les mutations de GATA2 et SBDS sont associées à des insuffisances médullaires prédisposant à des cancers myéloïdes. Une mutation de SBDS est reliée à une survie courte des patients après transplantation. Les mutations de GATA2 et PIGA sont en revanche des facteurs pronostiques favorables chez les jeunes adultes. Lorsque la mutation SBDS est bi-allélique, elle est associée à des mutations somatiques de TP53, expliquant la faible survie des patients, ce qui suggère que les mutations de TP53 favorisent la progression des SMD.

Intégration des données cliniques et des données génétiques

Une analyse de partitionnement récursif $^{4}$ a permis d'identifier six sousgroupes de patients SMD en fonction de leurs données cliniques et génétiques (Figure 1). La présence d'une mutation de TP53 se révèle être la variable la plus importante et correspond au sousgroupe de patients avec la moins bonne survie après transplantation $(0,7 \mathrm{an})$ et le plus haut taux de rechute.

Parmi les patients âgés de plus de 40 ans dont le gène TP53 n'est pas muté, trois sous-groupes ont été identifiés. Deux présentent une courte survie

\footnotetext{
${ }^{4}$ Les méthodes de partitionnement récursif constituent le fondement des arbres de décision. Les arbres de décision peuvent s'appréhender comme une série de questions, dont les réponses vont permettre de constituer des groupes d'individus de plus en plus homogènes, jusqu'aux nœuds terminaux.
} 
après transplantation, avec soit des mutations de la voie de signalisation Ras, associées à une faible survie $(0,9$ an) et à un plus fort risque de rechute ; soit des mutations de JAK2, également associées à une faible survie ( 0,5 an) mais sans rechute. En l'absence de ces mutations, la survie des patients SMD de plus de 40 ans après transplantation est beaucoup plus élevée (2,3 ans). La présence des mutations de TP53 et JAK2, dans les cellules du sang périphérique de patients atteints de cancers non hématopoïétiques, montre qu'elles sont également associées à l'âge [8]. Parmi les patients âgés de moins de 40 ans sans mutation de TP53, deux sous-groupes ont également été identifiés en fonction de facteurs de risque cliniques: SMD secondaires, nombre de plaquettes, pourcentage de blastes dans la moelle au diagnostic, etc. La présence de facteurs de risque chez ces patients entraîne une médiane de survie à 2,3 ans avec un fort risque de décès sans rechute, alors que les patients ne présentant pas de facteur de risque ont un taux de survie à 3 ans de $82 \%$.

\section{Analyse de la survie en fonction du type de conditionnement prétransplantation}

L'intensité du conditionnement prégreffe est directement associée, dans cette étude, aux risques de rechute et à la survie du patient. Un conditionnement myéloablatif est associé à moins de rechutes après transplantation, mais à une mortalité plus élevée. Un conditionnement non myéloablatif est associé à un plus faible taux de décès, mais à un plus fort taux de rechutes. Pour les patients ayant des mutations de TP53 et/ou de JAK2, le type de conditionnement n'influence ni la survie du patient, ni les risques de rechute. Au contraire, pour les patients ayant des mutations touchant la voie de signalisation Ras, un conditionnement non myéloablatif augmente les risques de rechute à $l$ an (incidence cumulative à $42 \%$ versus $20 \%$ en absence de mutations de la voie Ras).

\section{Conclusion}

Les mutations génétiques sont un des facteurs majeurs reconnus actuellement pour mesurer la progression des SMD. Cette étude définit leur place dans le contexte de la transplantation allogénique de CSH chez les patients SMD. Selon le modèle développé ici, le séquençage d'une série de gènes avant une transplantation allogénique de CSH permettrait de prévoir la survie de ces patients (voir Figure 1) après la transplantation et en fonction de la nature du conditionnement pré-greffe. $\diamond$

Towards a personalized pre-

transplantation conditioning in patients with myelodysplastic syndromes

\section{LIENS D’INTÉRÊT}

Les auteurs déclarent n'avoir aucun lien d'intérêt concernant les données publiées dans cet article.

\section{RÉFÉRENCES}

1. Hamblin TJ. The myelodysplastic syndromes: pathobiology and clinical management. In : Bennett JM, ed. Epidemiology of the myelodysplastic syndromes The myelodisolastic syndromes: pathobiology and clinical management. Boca Raton (FL) : CRC Press, $2002: 16 \mathrm{p}$.

2. Nimer SD. MDS: a stem cell disorder but what exactly is wrong with the primitive hematopoietic cells in this disease? Hematology 2008 ; $1: 43-51$.

3. Bhatia R, Deeg HJ. Treatment-related myelodysplastic syndrome: molecular characteristics and therapy. Curr Opin Hematol $2011 ; 18: 77-82$.

4. Lindsley R, Saber W, Mar BG, et al. Prognostic mutations in myelodysplastic syndrome after stemcell transplantation. N Engl J Med 2017 ; 376 : 536-47.

5. Zhang L, McGraw KL, Sallman DA, List AF. The role of $p 53$ in myelodysplastic syndromes and acute myeloid leukemia: molecular aspects and clinical implications. Leuk Lymphoma 2017 ; 58 : 1777-90.

6. Mahfoudhi $\varepsilon$, Secardin L, Scourzic L et al. Propriétés et rôles biologiques des protéines TET au cours du développement et de l'hématopoïèse. Med Sci (Paris) $2015 ; 31: 268-74$.

7. Dujardin G, Daguenet $\varepsilon$, Bernard DG, et al. L'épissage des ARN pré-messagers : quand le splicéosome perd pied. Med Sci (Paris) 2016 ; 32 : 1103-10.

8. Ganguly BB, Kadam NN. Mutations of myelodysplastic syndromes (MDS): an update. Mutat Res Rev Mutat Res $2016 ; 769: 47-62$.
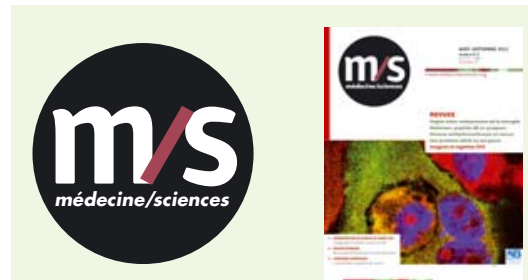

Abonnez-vous

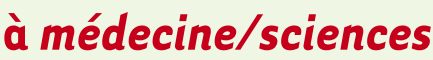

Bulletin d'abonnement page 98 dans ce numéro de $\mathrm{m} / \mathrm{s}$

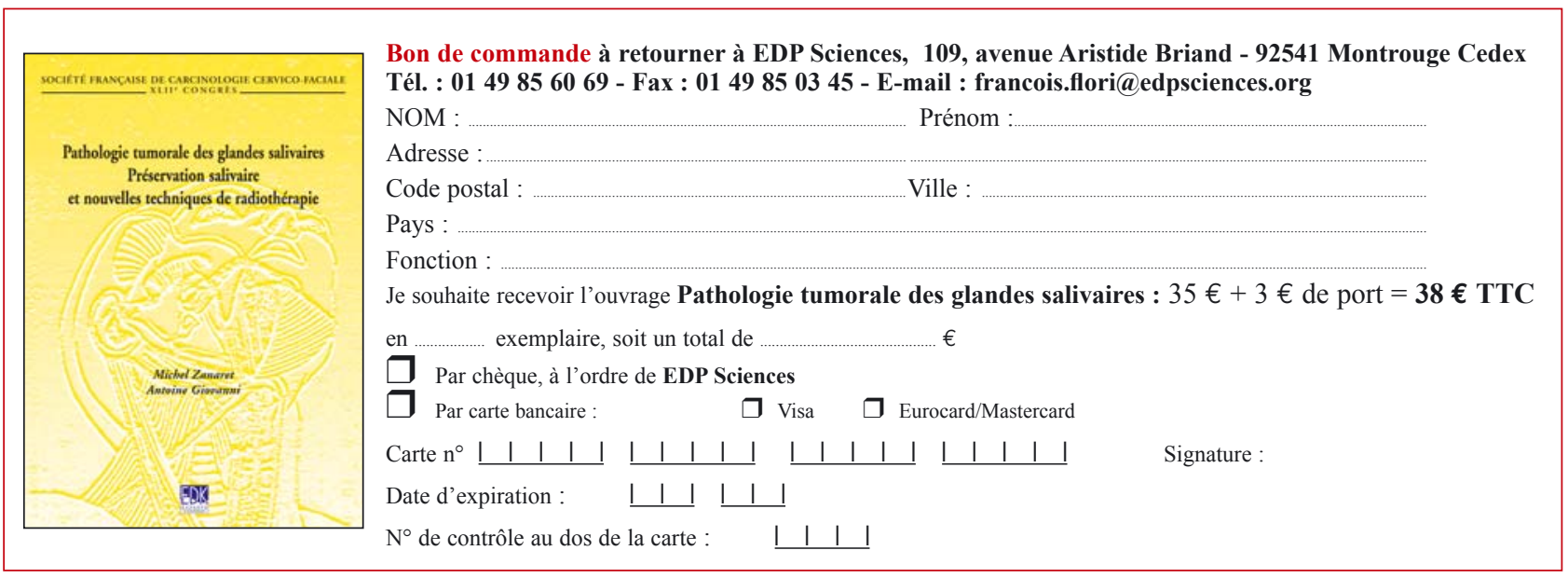

